NASA Technical Memorandum 100836

AIAA-88-0061

.. $r$.

\title{
Time-Accurate Simulations of a Shear Layer Forced at a Single Frequency
}

R.W. Claus

Lewis Research Center

Cleveland, Ohio

P.G. Huang

Michigan Technological University

Houghton, Michigan

and

J.M. MacInnes

Princeton University

Princeton, New Jersey

Prepared for the

26th Aerospace Sciences Meeting

sponsored by the American Institute of Aeronautics and Astronautics

Reno, Nevada, January 11-14, 1988

\section{N/SA}

(NASA-TM-100836) TIME-ACCUEATE SIMULATIONS

OF A SHEAR LAYER FORCED AT A SINGLE

FRE QUENCY (NASA) 20 P

$\operatorname{Csc} 2$ 2OD 


\title{
TIME-ACCURATE SIMULATIONS OF A SHEAR LAYER FORCED AT A SINGLE FREQUENCY
}

\author{
R. W. Claus, NASA Lewis Research Center \\ P. G. Huang, Michigan Technological University \\ and J. M. MacInnes, Princeton University
}

\begin{abstract}
This report presents calculations of the forced shear layer studied experimentally by Oster and Wygnanski (ref. 1) and Weisbrot (ref.2). Two different computational approaches are examined: Direct Numerical Simulation (DNS) and Large Eddy Simulation (LES).The DNS approach solves the full three-dimensional, Navier-Stokes equations for a temporally-evolving mixing layer, while the LES approach (as used in this report) solves the two-dimensional, Navier-Stokes equations with a subgrid scale turbulence model. While the comparison between these calculations and experimental data was hampered by a lack of information on the inflow boundary conditions the calcuations are shown to agree qualitatively with several aspects of the experiment. The sensitivity of these calculations to factors such as mesh refinement and Reynolds number is illustrated.
\end{abstract}

NOMENCLATURE

$$
\begin{aligned}
& \lambda=\text { forcing wavelength } \\
& \delta_{0 . x 0}=\text { radial location where the flow reaches } x \text { percent of } U_{\infty}
\end{aligned}
$$

\section{INTRODUCTION}

Great interest has recently been directed toward the study of forced turbulent shear flows. Practically, the concern is with controlling the flow in order to achieve various desired results such as increased mixing. The hope is that through a knowlegable application of very low amplitude forcing (i.e. low energy input) one can significantly alter the flow development such that a large-amplitude effect is achieved. Currently our understanding of how these processes occur and how to manipulate them is increasing dramatically, but significant questions remain. To truely tap the potential of these processes requires an understanding of the basic physics of the flow field. Specifically, the interaction of vorticies of various scales must be more thoroughly understood. 
An excellent example of a flow field in which a wide range of vortices interact is the experiment of Oster and Wygnanski (ref. 1) and later Weisbrot (ref. 2). In this experiment a well-organized, periodic, large-scale motion is produced by forcing at a single frequency. This structure evolves and develops in a turbulent flow extracting energy from, and later imparting energy to, the mean flow. The manner in which these large-scale structures interact with what is more commonly referred to as turbulence (small scale, random motions) yields a great deal of information on flow dynamics and provides a strong challenge to those interested in calculating these flows.

In this report various numerical methods are examined in an attempt to numerically simulate the experiment of ref. 2. One computational approach solves the two-dimensional, time-dependent equations of motion with a two equation turbulence model. This approach, defined as a Large Eddy Simulation (LES) in this report, assumes that the large scale fluctuations in a shear layer are mainly twodimensional and that the three-dimensional, random motions can be represented by a turbulence model. Two dimensional, laminar flow calculations are also presented - to compare and contrast with the LES calculations. The final computational approach examined is Direct Numerical Simulation (DNS). With this approach the three-dimensional, Navier-Stokes equations are solved for a temporally evolving shear layer. Each of these various computational approaches has unique advantages and disadvantages. The purpose of this report is to examine whether, in ensemble, these computations can predict the major features of the excited shear layer.

\section{Computational Approach}

\section{Large Eddy Simulations}

Several different computational approaches toward making time-accurate calculations are employed in this report. The first approach, referred to here as a Large Eddy Simulations (LES), solves the two-dimensional, Navier-Stokes equations with a two-equation turbulence model. The turbulence model is used to represent the non-periodic motion while the large scale motions are captured on a computational mesh. This methodology follows the Deardorff approach which recognizes that the solution of the discretized equations using a finite volume algorithm is mathematically equivalent to solving the original equations with a "box" type filter, ref. 3. The advantage of this approach is that the sub-grid scale turbulence model is significantly simpified. The (Leonard) stresses that arise through the use of a more general filter (for example- a Gaussian filter) are zero. This permits the use of a turbulence model that is simply a time-dependent form of the commonly-used, 
Reynolds-averaged, $k-\epsilon$ model, ref.4. There are some disadvantages to this approach and reference 5 is recommended as a source detailling these issues. It is sufficient to state that, at this time, it is not clear that any significant penalties are incurred through the use of this approach.

The numerical scheme used to solve the discretized equations is an implicit, incompressible flow algorithm. Flow variables are represented on a staggered-mesh with Crank-Nicholson time differencing and QUICK differencing (ref. 6) of the convective terms. This maintains second order accuracy in both time and space. Continuity is enforced through the iterative pressure-correction scheme SIMPLE (Semi-Implicit Pressure Linked Equations), ref.7. This code is described in detail by its originators in ref. 8 and the code has undergone extensive testing and evaluation in idealized benchmark problems, ref. 9, and numerous practical flow calculations. Modifications were made to the code to improve vectorization and a more efficient solver, employing the Stone's strongly implicit algorithm combined with a blockcorrection, is used to solve the pressure-correction equation, ref. 10.

In applying this code to the time-dependent shear layer calculations, the domain extends from the transverse plane which is just at the trailing edge of the splitter plate (figure 1) and extends the full length of the experimental test section. Boundary conditions for $\mathrm{u}, \mathrm{v}, k$ and $\epsilon$ at the inlet are prescribed according to estimates of the experimental conditions. The upper and lower boundaries were treated as imposed symmetry conditions. Entrainment conditions are used at the flow exit, which amounts to fixing pressure, employing cell mass balance to obtain $u$ and applying either a zero gradient condition for other variables when the flow is out of the domain or, when the flow is inward, the values for each quantity must be known and a fixed boundary condition is used.

The strongest gradients in these calculations occur in the central region of the flow domain and have a dominant $y$ - direction component. To accomodate these gradients one-third of the mesh points in the y direction are uniformly distributed in the central $10 \%$ of the domain. The remaining two-thirds are divided among the upper and lower portions with variable spacing so that a smooth transition is made at the interface. For the fine mesh calculations ( $798 \times 241$ grid points), grid point spacing was expanded 0.1 percent per grid cell in the axial direction. The expansion factor for the coarse mesh calculations $(150 \times 80)$ was 0.267 percent and the expansion factor for the $400 \times 241$ mesh calculations was 0.2 percent. These mesh points were distributed over a range of $0-2000 \mathrm{~mm}$ in the $\mathrm{X}$ direction and -200 to $+200 \mathrm{~mm}$ in the $\mathrm{Y}$ direction.

The shear layer is known as a convectively unstable type of flow, ref. 11 This means that small perturbations upstream grow exponentially as they are convected with the flow. For this reason, it was recognized that the treatment of the inflow boundary condition was very important in establishing a correct comparison with experimental data. Unfortunately, detailed measurements near the splitter plate 
were not available so that it was neccessary to estimate the thickness of the boundary layers as they left the splitter plate. A momentum thickness $(\theta)$ estimate of $0.5 \mathrm{~mm}$ for the layer on each side of the splitter plate was inserted into an exponential profile:

$$
u=u_{\infty} \exp (-y / \delta), \delta=2 \theta
$$

This profile was then oscillated in the y direction at the frequency and amplitude corresponding to experimental conditions. Various modifications to this boundary profile were tested, and without examining an infinite number of permutations, this profile was selected as reasonably representative of experimental conditions. But it is certainly true that the calculations are very sensitive to this boundary condition and it is appropriate here to emphasize the importance of documenting the details of the inlet flow for an experiment to provide useful information for testing calculations.

Inlet conditions for the turbulence model were somewhat easier to establish. The experiment documented a streamwise turbulence intensity of around 0.2 percent, so $k \simeq \overline{u^{\prime 2}}=4 x 10^{-4} \mathrm{~m}^{2} / \mathrm{s}^{2}$ is used. The length scale of the turbulence entering the test section was not documented, but can be estimated from the formula $\epsilon=k^{3 / 2} / l$ using a length scale on the order of $l \simeq 2 \mathrm{~mm}$. Fortunately, some test calculations indicate that the results are not sensitive to the precise value of $\epsilon$ used, within a reasonable range.

As noted previously, these are incompressible flow calculations wherein a perturbation anywhere in the flow field can be "felt" everywhere else in the flow. Practically, what this requires is that the calculations be run for a long time period to allow the initinial conditions of the flow to be completely "flushed" through the computational domain until periodic flow behavior is reached. This is partially affected by the level of mass residual allowed in the iterative calculation, where the lower the residual, the faster the approach to periodicity. The laminar flow calculations were much more sensitive to this effect and generally had to be run for longer flow times to reach periodicity.

\section{Laminar Flow Calculations}

These calculations are essentially the same as those noted above with the exception of the turbulence model. In these calculations, only a constant molecular viscosity is used. This laminar viscosity is not intended as a subgrid scale turbulence model, but rather to constrast the effect of the two-equation model on the time-accuate calculations. The Reynolds number based on the mean convective velocity and the wavelength of forcing was 96000 .

\section{Direct Numerical Simulations}

The term, Direct Numerical Simulation, as used in this report refers to the solution of the three-dimensional, time-dependent Navier-Stokes equations by a highly 
accurate numerical scheme such that all the scales of motion are well-resolved on a computational mesh. This imposes two major restrictions on this computational scheme. First, although highly accurate spectral methods are used to solve the governing equations, only a limited range of fluctuating motions can be resolved on the computational mesh. This restricts the calculations to a low Reynolds number flow (approximately 50 to 100 based on the Taylor microscale). When one applies the results of a low Reynolds number calculation to understand high Reynolds number turbulence, an inherent assumption is that the large energetic scales of turbulent motion display characteristics that are Reynolds number independent. The second major limitation of these Direct Numerical Simulations, is that the use of spectral methods (Fourier series) imposes the need to use periodic boundary conditions in the main flow direction. This means that these calculations are of a temporally-evolving shear layer as opposed to the spatially-evolving experiment. This comprises a Lagrangian description of the spatially developing shear layer with the computational domain following the mean flow. The drawback that this imposes in comparisons with experimental data is offset by the increased numerical resolution available in the Lagrangian description of the flow. These time-evolving simulations should be looked at as an "idealization" of the real flow.

In comparing these temporal simulations to spatial experimental data, a transformation of the form $X=X_{0}+U_{c} t$ must be performed. Where $U_{c}$ is the convective velocity of the large eddies. The choice of $X_{0}$ is somewhat arbitrary, so this was chosen to yield agreement with the first experimental data point documented in ref. 2 and then held constant for the rest of the data. This issue is also discussed in ref. 12.

\section{RESULTS AND DISCUSSION}

A schematic outline of the flow geometry and the main features of the forced, flow field are illustrated in figure 1. Intinially the shear layer is subject to very rapid growth due to the roll-up of vorticies scaling on the wavelength of the forcing. Somewhat further downstream, the roll-up process saturates and the shear layer stops growing. This is followed by a collapse of the layer width and then by a slow, secondary growth. These are some of the main characteristics of the flow field, documented in ref. 2 , that will be compared against the numerical simulations.

The results of two-dimensional, laminar flow calculations are displayed in figure 2. In general, the calculations display the development of both positive and negative vorticity as the boundary layers leave the splitter plate and form large scale structures. The positive vorticity regions (dashed contours) result from the low speed boundary layer that is used as an inflow boundary condition. These are 
rapidly dissipated. The mean flow gradient encourages the formation of negative vorticity, and the calculations indicate that this is the main component of vorticity in the flow field.

The effect of grid resolution can be seen in the instantaneous vorticity contours in figures $2 \mathrm{a}$ and $2 \mathrm{~b}$. The coarse mesh calculation initially forms two small discrete vorticies that rapidly coalsce into one larger vortex, scaling on the wavelength of the periodic forcing. The fine mesh calculation initially forms many more smaller scale vorticies and approximately six of these vorticies slowly coalesce and merge into a single vortex structure very near the exit of the computational domain. The fine mesh calculation retains much higher levels of negative vorticity throughout the flow field.

These marked differences between the fine and coarse mesh calculations indicate that the calcuations are not mesh independent. For at least the laminar flow calculations, it appears that mesh refinement yields very small scale detail that can significantly affect the development of the flow. Even the high level of mesh refinement present in the $798 \times 241$ mesh calculation is probably insufficient to fully resolve this flow field. The inflow boundary conditions impose a point discontinuity in the velocity profile, providing a continuous source of ever smaller scale vorticity. The actual experimental values may not be this severe, but lacking experimental data, a definitive evaluation can not be made.

The Reynolds stress field averaged over one cycle of the imposed forcing is displayed in figure 2c. The dashed lines indicate regions of negative Reynolds stress where the energy transfer is from the fluctuating motions to the mean flow: the so-called "inverse cascading" seen in forced flows. Small regions of negative stress appear first on the low speed side of the layer and then later on the high speed side. These small regions of negative stress are caused by individual, small vorticies changing their orientation with respect to the main flow. Downstream from these isolated pockets of negative stress, a large region of negative stress is formed due to the orientation of the whole vortex ensemble. This is the region where the layer undergoes a "collapse" and noticeably reduces in thickness. Downstream from the large region of negative stress, the flow alternates between positive and negative stress as the large scale, forced structure exhibits vortex nutation.

The LES calculations are significantly different from the laminar flow calculations as seen in figure 3. In general, the contours of vorticity display a much more organized (or smooth) flow pattern than the fine mesh, laminar flow calculations. The effective viscosity added by the turbulence model serves to damp-out most of the small scale features that form near the inflow boundary. The only remaining structure is the periodic structure associated with the forcing. This structure rapidly forms and then dissipates as it travels downstream.

Figures $3 a$ and $3 b$ display the results of LES calculations for two different mesh densities. These results differ less than the laminar comparison, but still indicate 
some grid dependency. It is interesting that the fine mesh calculation appears smoother than the coarse mesh calculation near the inflow boundary. Apparently, the reduction in numerical diffusion in the fine mesh calculation is more than offset by the increased turbulence generation produced by the model in response to sharper velocity gradients.

The averaged Reynolds stresses (evaluated from the oscillatory component of motion) are shown in figure 3c. These stresses are much more uniform than in the laminar flow calculations. A large region of positive stress, near the inflow boundary, changes to low levels of negative stress further downstream. About half way through the flow field, the stresses become negative and remain largely unchanged until near the exit. In this region of the flow, the effect of the turbulence model has been to diffuse vorticity and thereby slow down the interaction between the vorticity and the mean flow. This changes the rate at which the stresses change sign.

It should be remembered that the fluctuating motion in the LES calculations represents only part of the total turbulent kinetic energy in the calculation - the turbulence model supplies the rest. The magnitude of these two different components of the kinetic energy can be compared in the instantaneous profiles displayed in figures $3 \mathrm{~d}$ and $3 \mathrm{e}$. In general, the kinetic energy in the oscillatory motion is greater than that supplied by the turbulence model, especially in the middle of the flow domain. Near the inflow boundary, where mean gradients are quite steep, the turbulence model displays its highest levels of kinetic energy. Near the outflow boundary the kinetic energy associated with the turbulence model is of the same approximate level as that of the oscillatory motion. One interesting feature of the turbulence model kinetic energy is that local maximas are seen in regions of the flow commonly referred to as the braids. These are regions of intense shear that have been experimentally shown to be the source of counter-rotating, streamwise vorticies that are a major component of three-dimensional "random" turbulence, ref. 13. It is certainly encouraging that the turbulence model qualitatively represents this trend. To establish whether this effect is quantitatively correct would require more detailed experimental measurements.

A "close-up" comparison between these calculations and experimental data is shown in figures 4, 5 and 6 . Figure 4 compares the instantaneous vorticity contours* at axial locations around $200-560 \mathrm{~mm}$. In this region of the flow, near the end of the splitter plate, the initial vortex roll-up is visable. Experimentally, the initial vortex displays a number of vorticity maximas (perhaps three or four) and the next vortex (around $440 \mathrm{~mm}$ ) displays two maximas. The laminar flow calculation displays many more vorticity maximas and especially the structure of the second

* Contour levels were not documented in the experimental data. The computational contour levels were chosen to reveal the main features of the flow and should not be expected to display the same density of lines as in the experiment. 
vortex is markedly different. The LES calculation dissipates the small scale vorticies so that only a few maxima are seen (approximately one per structure). The LES calculation appears to show too little small scale structure while the laminar flow calculation shows too much.

Figure 5 displays the instantaneous vorticity contours around axial locations of $560-840 \mathrm{~mm}$. Again, as in the previous figure, the laminar flow calculation displays too much small scale structure. The LES calculation displays two, very smooth vorticies which is roughly in agreement with the experimental contours.

Figure 6 displays the instantaneous vorticity contours around axial locations of $840-1780 \mathrm{~mm}$. Again the laminar flow calculation displays too much small scale structure and, near the exit (around $1700 \mathrm{~mm}$ ), a double vortex structure is seen. In the LES calculations, the effect of dissipation can be seen through the decreasing density of the vorticity contours. A similar trend is seen in the experimental data with the exception that the large structure appears now to be composed of two, nearly vertically-alined, vorticies.

From these various "close-up" comparisons, it appears as though the LES calculation is initially too dissipative near the inflow boundary or close to the splitter plate. Further on, in the free-shear region of the flow, the turbulence model appears to provide approximately the correct level of dissipation. This suggests that some mechanism may be needed to modify the dissipation rate equation such that the behavior in the near wake of the boundary layer is less dissipative. Essentially, the length scale calculation near the splitter plate needs to be different from that in the free-shear region.

A comparison of calculated and experimentally measured mean axial velocity layer position as a function of downstream distance is shown in figure 7. Figure 7a compares the two-dimensional LES and laminar flow calculations. In general, both computations grow rapidly in the initial portion of the flow field and appear to saturate at approximately the same point as the experimental data. The laminar calculations reveal saturation of several, small vorticies leading to the "bumpy" nature of the velocity layer growth. The LES calculation exhibits a very smooth velocity layer growth, but the sharp collapse of the layer, seen experimentally, is not reproduced in the calculation. Neither of the calculations appear to indicate the slow secondary growth on the low velocity side.

Figure $7 \mathrm{~b}$ compares DNS calculations made at two different initial Reynolds numbers with the experimental data*. The low Reynolds number results ( $\mathrm{Re}=250$ ) compare quite favorably with the low speed side of the velocity profile. Unfortunately, the DNS results are symmetric about the centerline and cannot capture the

* The DNS calculations were for a temporally-evolving layer. To compare with experimental data required a transformation between temporal and spatial variables as noted earlier. 
difference between the high speed and the low speed sides of the layer. (It is not clear whether or not different initial conditions for these simulations might be able to pick up this asymmetric development of the layer. This remains a question for further research.) The higher Reynolds number results $(\mathrm{Re}=500)$ are very similar to the low Reynolds number results except for near the end of the computations. These simulations exhibit a very strong growth rate instead of the slow, secondary growth, documented in the experiment. As will be shown in the next figure, this region of very strong growth is due largely to three-dimensional streamwise vorticies that lead to cascading energy towards smaller scales. The energy built up at these smaller scales cannot be resolved on the computational mesh and, shortly after the period displayed in figure $7 \mathrm{~b}$, the calculation becomes unstable. This is a characteristic weakness of the DNS approach (as noted earlier), but the trends exhibited by the calculation should be valid. Fine-tuned initial conditions and a much finer mesh resolution might lead to improved agreement with experimental data.

The energy content of various modes in the DNS calculations is shown in figure 8 for the two different Reynolds numbers. The fundamental mode (the excited wave) and the $3 \mathrm{D}$ modes (streamwise vorticies) are the primary structures in this flow field. The fundamental mode grows rapidly early in the calculation and is largely unaffected by Reynolds number. Around a time of 10 seconds (or $600 \mathrm{~mm}$ ) the fundamental mode saturates and then, generally, loses energy. In this region of energy loss for the fundamental mode, the 3D modes gain energy very rapidly. This trend is strongly affected by Reynolds number. Essentially what is occuring is that the $2 \mathrm{D}$ spanwise structure ( the fundamental mode) is strongly stretching the $3 \mathrm{D}$ conter-rotating vorticies that form in the braid region of the flow. This feature of the flow is graphically documented in ref. 12. This growth of the streamwise structures causes a growth in the mean thickness of the shear layer.

A comparison of calculated and experimentally measured Reynolds stresses is shown in figure 9. The various computational approaches display very different trends. In general, none of the calculations are quantitatively correct, although the DNS and the LES calculations always display the appropriate trend (or sign). The LES stresses are a combination of the stresses contributed by the oscillatory motion and the turbulence model. The turbulence model does not significantly contribute to the Reynolds stresses at $x=440 \mathrm{~mm}$ or $x=720 \mathrm{~mm}$, but it is significant enough at $x=1700 \mathrm{~mm}$ to change the sign of the stresses from negative to positive. The LES calculations very closely match the experimental data at $x=440 \mathrm{~mm}$, but are in poorer agreement with the measurements downstream. The laminar calculations approximate the data at $x=440 \mathrm{~mm}$, but again are in poorer agreement further downstream. The worst agreement is exhibited at $x=1700 \mathrm{~mm}$ by the laminar calculations where the sign of the stresses is incorrect.

The experimental data also include information on the Reynolds stresses contributed by the large forced structure. This information was obtained by taking 
measurements that were "phase-locked" on the frequency of forcing. The stresses contributed by the forced structure could then be separated from the total stresses that would be obtained from a long time-average. Figure 10 displays the "phasedlocked" and total stresses measured experimentally at $x=1700 \mathrm{~mm}$. These results are compared with the LES and DNS calculations. For the DNS calculations, the total stresses are presented for two different Reynolds numbers. The main difference in these two calculations is the strength of the 3D modes (or the streamwise vorticies) as seen in figure 8. At the higher Reynolds number the streamwise vorticies are strong enough to cause the stresses to be significantly positive. At the lower Reynolds number the stresses are so small as to effectively be zero across the layer. The LES calculations indicate that the stresses due to the oscillatory motion are negative, while the total stresses (a combination of the oscillatory motion stresses and the turbulence model stresses) are positive. While the LES results are in qualitative agreement with the experimental results, the magnitude of the calculated stresses is much smaller than the experimental values.

\section{Concluding Remarks}

At this point it is appropriate to restate the question implicitly posed the introduction to this report: In ensemble, can the various computational approaches examined predict the major features of the excited shear layer? Lacking detailed measurements of the inflow boundary conditions, it is impossible to definitively answer this question. Certainly the initial growth and saturation of the shear layer are approximated by all calculations. The laminar and the LES calculations display very different small-scale behaviour, but the initial roll-up is largely dominated by the forced structure and all the calculations represent this feature. From this point in the flow, however, the DNS calculations indicate that small-scale, streamwise vorticies grow very rapidly. This transition from two-dimensional forced motion to three-dimensional and, eventually, rather random motion seems likely to be the cause of the slow secondary growth found experimentally. The LES calculations do display increased levels of turbulence kinetic energy in the braid regions where the streamwise structures originate, but this has no significant effect on the mean flow. The oscillatory motion dominates the stresses in the LES calculations up until very near the outflow. Near the outflow, the turbulence model significantly alters the stresses such that the negative stresses contributed by the oscillatory structure are made positive. Although this trend is in agreement with the experimental data, it is quantitatively too small to significantly affect the mean velocity profile and display some secondary growth. The DNS calculations may over-estimate the importance of the streamwise vorticies due to its inability to cascade energy to scales both larger than and smaller than the computational mesh. 
The final point to be emphasized is the importance of mesh refinement. Although new supercomputers have made possible the fine mesh calculations reported here, it is likely that these results (at least for laminar flow) remain grid dependent. This grid dependency can strongly alter the calculated vorticity structure and the development of the flow.

\section{Acknowledgements}

The authors are listed alphabetically and the use of the NAS Cray 2 for all of these calculations is gratefully acknowledged.

\section{References}

[1] Oster, D. and Wygnanski, I., J. of Fluid Mech., 1982, vol. 124, 91-130.

[2] Weisbrot, I., "A Highly Excited Turbulent Mixing Layer," M.S. Thesis, Tel Aviv University, Dept. of Fluid Mechanics and Heat Transfer, 1984.

[3] Deardorff, J. W., "A Numerical Study of Three-Dimensional Turbulent Channel Flow at Large Reynolds Number," 1970, J. of Fluid Mech., vol. 41, 452-480.

[4] Launder, B. E. and Spalding, D. B., "The numerical computation of turbulent flows," Computer Methods in Applied Mechanics and Engineering, 3, 269-289 (1974).

[5] Ferziger, J. H., "Higher level simulations of turbulent flows," in Computational Methods for Turbulent, Transonic, and Viscous Flows, Hemisphere Publishing, 1983.

[6] Leonard, B. P.: Comp. Methods in Applied Mech. and Eng. 19, 59 (1979).

[7] Patankar, S. V. and Spalding, D. B., " A calculation procedure for heat, mass and momentum transfer in three-dimensional parabolic flows," International Journal of Heat and Mass Transfer, Vol. 15, No. 10, Oct. 1972, pp. 1787-1806.

[8] Huang, P. G. and Leschziner, M. A., Thermofluids Division technical report TFD/83/9(r), Department of Mechanical Engineering, UMIST.

[9] Huang, P. G., Launder, B. E. and Leschziner, M. A., Comp. Methods in Applied Mech. and Eng., Vol. 48, 1985.

[10] Turan, A. and VanDoormal, J. P., "Improved numerical methods for turbulent viscous recirculating flows," NASA CR 180852, 1987.

[11] Bechert, D. W., "Excitation of Instability Waves," Z. Flugwiss. Weltraumforsch. 9 (1985), Heft 6.

[12] Claus, R. W., " Direct Numerical Simulations of a Temporally Evolving Mixing Layer Subject to Forcing," NASA TM 88896, 1986. 
[13] Hussain, A.K.M.F. and Zaman, K.B.M.Q., "An experimental study of organized motions in the turbulent plane mixing layer", JFM, vol. 159, 1985, pp. 85-104.

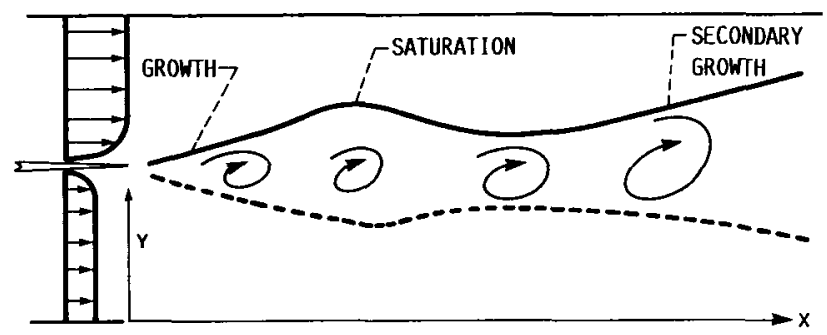

FIGURE 1. - TWO-DIMENSIONAL FLOW GEOMETRY SHOWING THE ORIENTATION OF THE AXES AND MAJOR FLOW FIELD FEATURES.

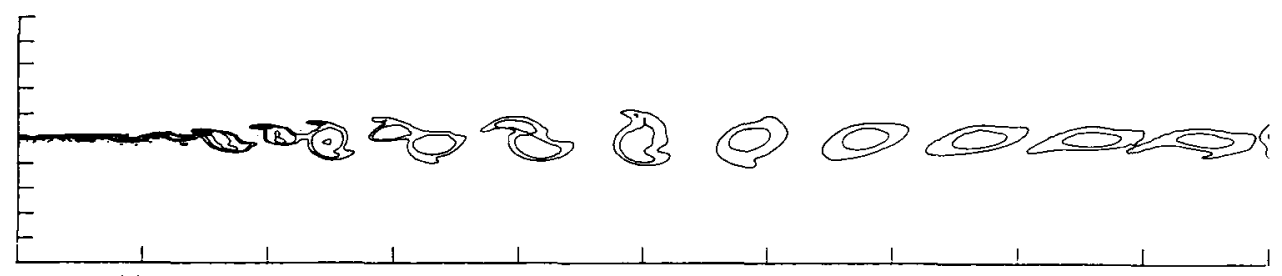

(A) INSTANTANEOUS VORTICITY FOR A COARSE MESH CALCULATION (150 $\frac{1}{1} \frac{1}{1}$ $-500,-250,-100,-50$. AND DASHED $+50,+100$.

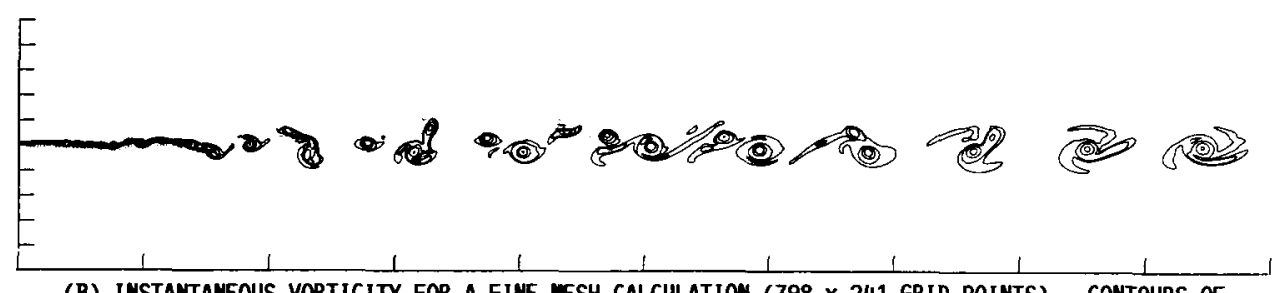

(B) INSTANTANEOUS VORTICITY FOR A FINE NESH CALCULATION (798 $\times 241$ GRID POINTS), CONTOURS OF $-500,-250,-100,-50$. AMD DASHED $+50,+100$.

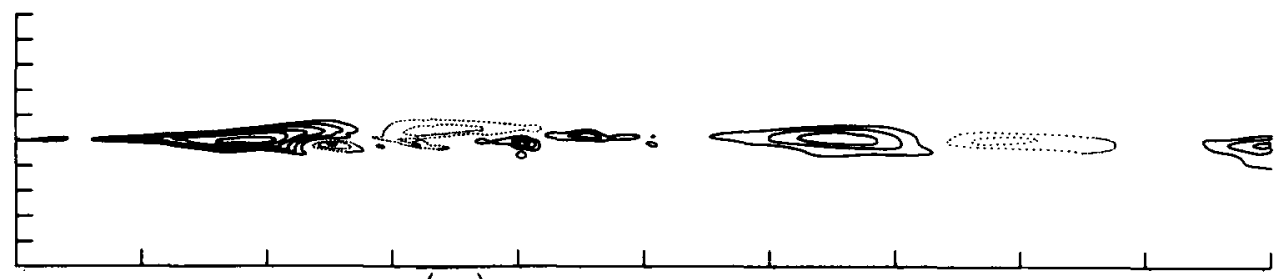

(C) AVERAGED REYMOLDS STRESSES $\left(\overline{\frac{-U V}{\Delta U^{2}}}\right)$ OF THE OSCILLATORY MOTION (798 $\times 241$ GRID POINTS). CONTOURS RANGE FROM: SOLID LIMES, 0.0150 TO 0.0025 IN EQUAL IMCREMENTS AND DASHED LIMES, -0.0075 TO 0.0025 IN EQUAL IMCREMENTS.

FIGURE 2. - COMPUTATIOHAL RESULTS FOR THE TWO-DIFENSIONAL LAMIMAR FLOW CALCULATIONS. 


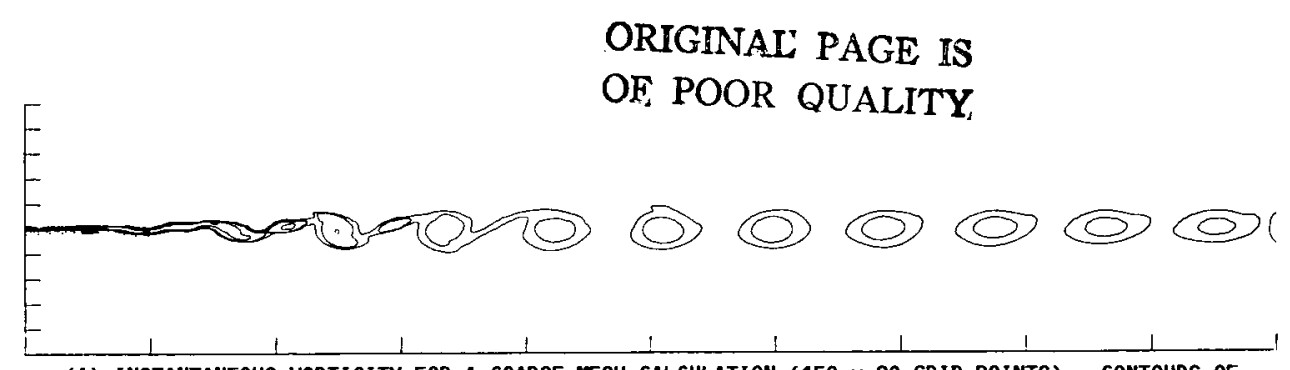

(A) INSTANTANEOUS VORTICITY FOR A COARSE MESH CALCULATION (150 x 80 GRID POINTS). CONTOURS OF $-500,-250,-100,-50$, AND DASHED $+50,+100$.

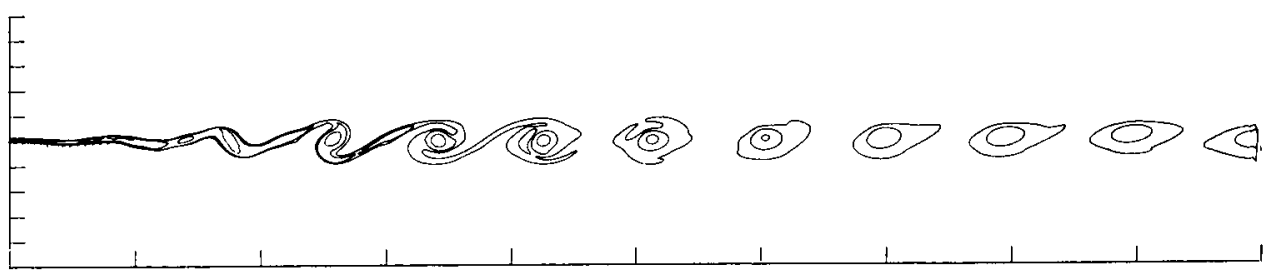

(B) INSTANTANEOUS VORTICITY FOR A FINE MESH CALCULATION ( $400 \times 241$ GRID POINTS). CONTOURS OF $-500,-250,-100,-50$, AND DASHED $+50,+100$.

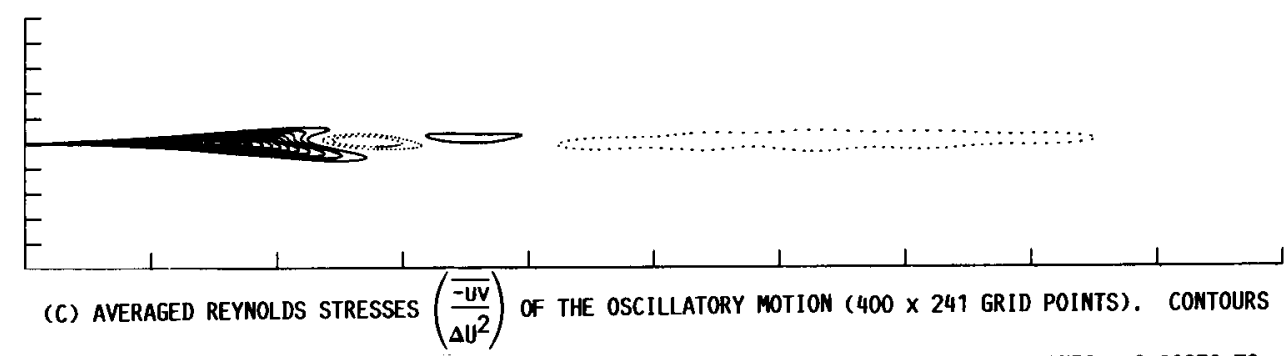

RANGE FROM: SOLID LINES, 0.01250 TO 0.00125 IN EQUAL INCREMENTS AND DASHED LINES, -0.00250 TO -0.00125 IN EQUAL INCREMENTS.

$E$

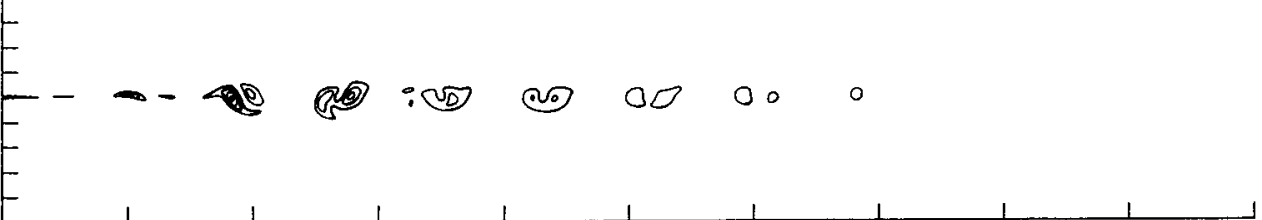

(D) INSTANTANEOUS KINETIC ENERGY OF THE OSCILLATORY MOTION ( $K=3.0$ TO 0.5 INTERVALS OF 0.5 ).

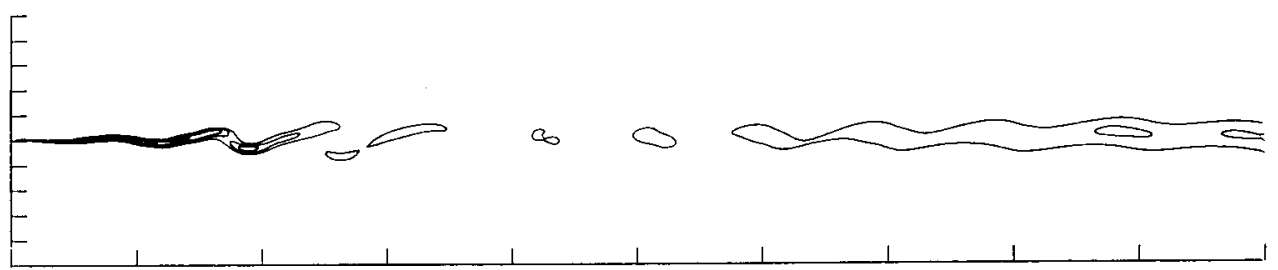

(E) INSTANTANEOUS TURBULENCE KINETIC EMERGY FROM THE TURBULENCE MODEL ( $K=0.7$ TO 0.1 EQUAL INTERVALS). FIGURE 3. - COMPUTATIONAL RESULTS OF THE LES CALCULATIONS ( $400 \times 241$ GRID POINTS UNLESS NOTED). 


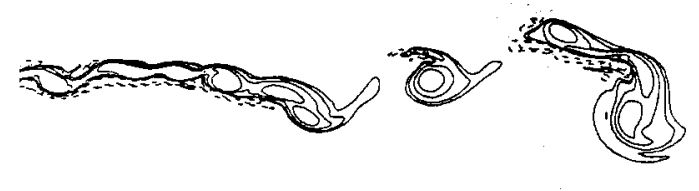

(A) LAMIMAR CALCULATIONS (798 $\times 241$ GRID POINTS).

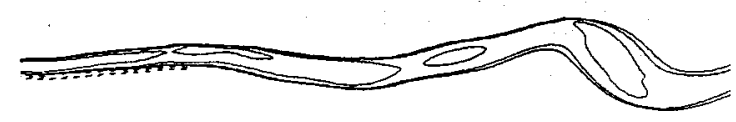

(B) LES CALCUALTIONS ( $400 \times 241$ GRID POINTS).

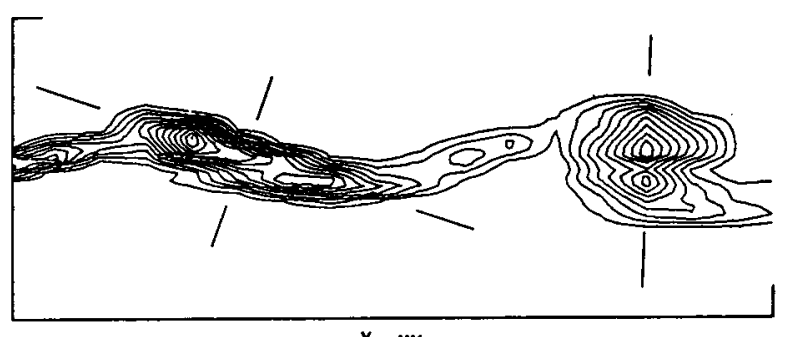

$x, m$

(C) EXPERIMENT.

FIGURE 4. - INSTANTANEOUS VORTICITY CONTOURS FOR THE TWODIMENSIONAL CALCULATIONS AND EXPERIMENTAL DATA AROUND AXIAL LOCATIONS OF 200 TO $560 \mathrm{~mm}$.
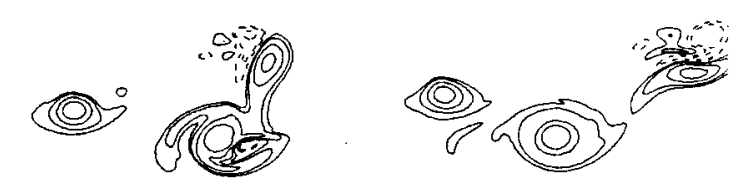

(A) LAMINAR CALCULATION (798 $\times 241$ GRID POINTS).

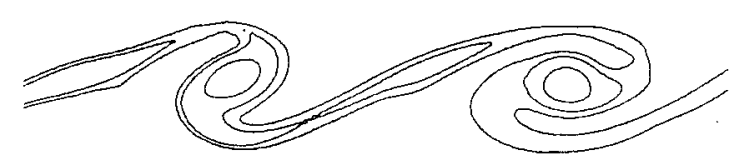

(B) LES CALCULATION ( $400 \times 241$ GRID POINTS).

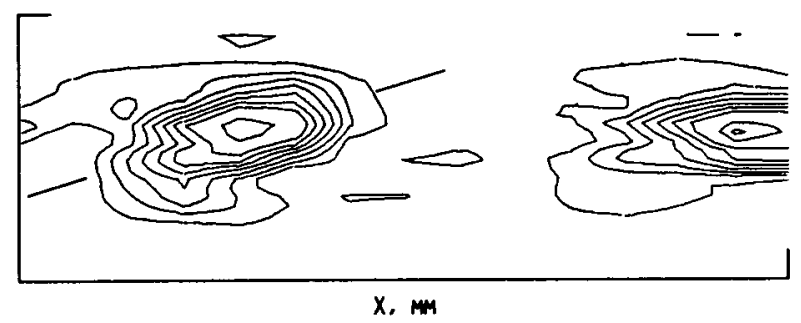

(C) EXPERIMENT.

FIGURE 5. - INSTANTANEOUS VORTICITY CONTOURS FOR THE TWODIMENSIONAL CALCULATIONS AND EXPERIMENTAL DATA AROUND AXIAL LOCATIONS OF 560 TO $840 \mathrm{~mm}$. 


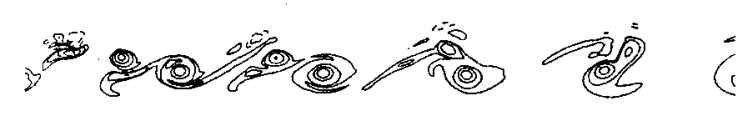

(A) LAMINAR CALCULATION (798 × 241 GRID POINTS).

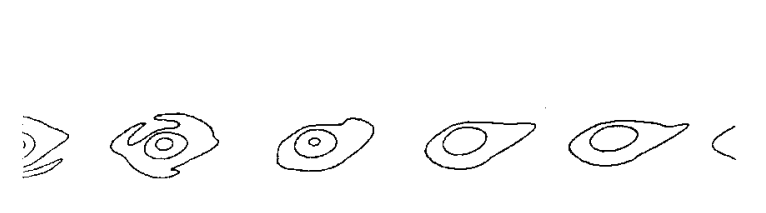

(B) LES CALCULATIONS ( $400 \times 241$ GRID POINTS).

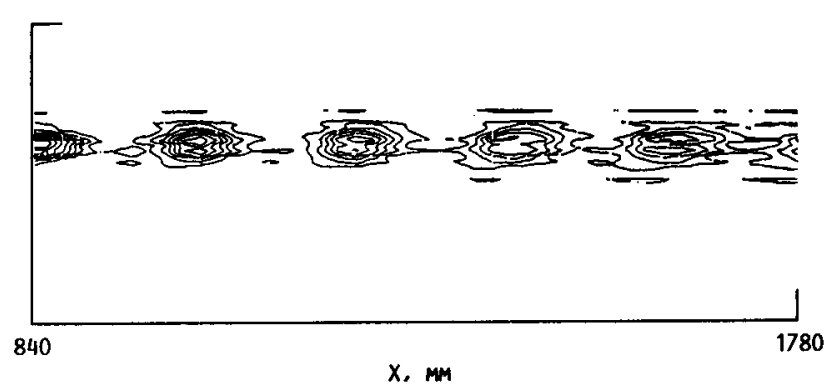

(C) EXPERIMENT.

FIGURE 6. - INSTANTANEOUS VORTICITY CONTOURS FOR THE TWODIMENSIONAL CALCULATIONS AND EXPERIMENTAL DATA AROUND AXIAL LOCATIONS OF 840 TO $1780 \mathrm{mM}$.

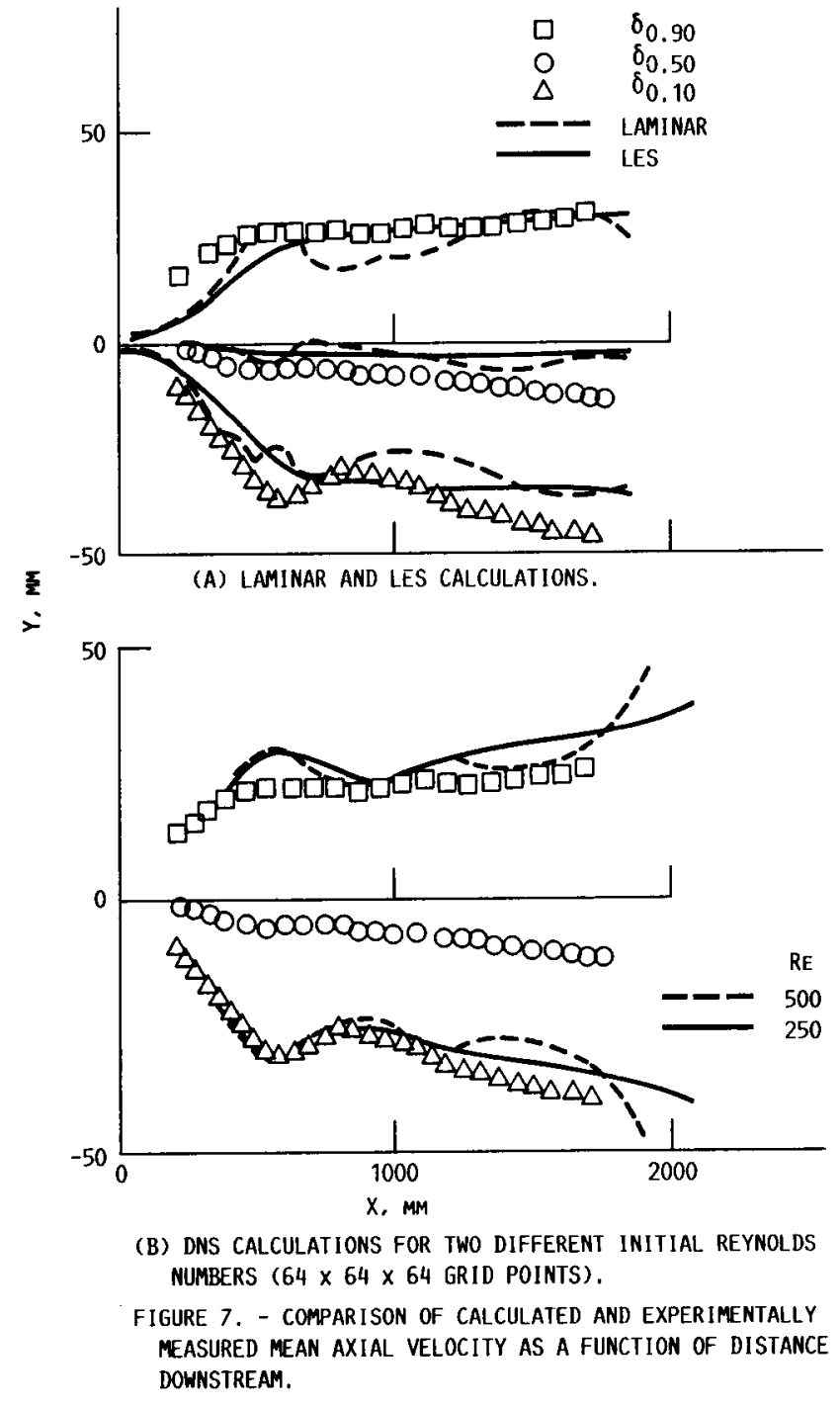




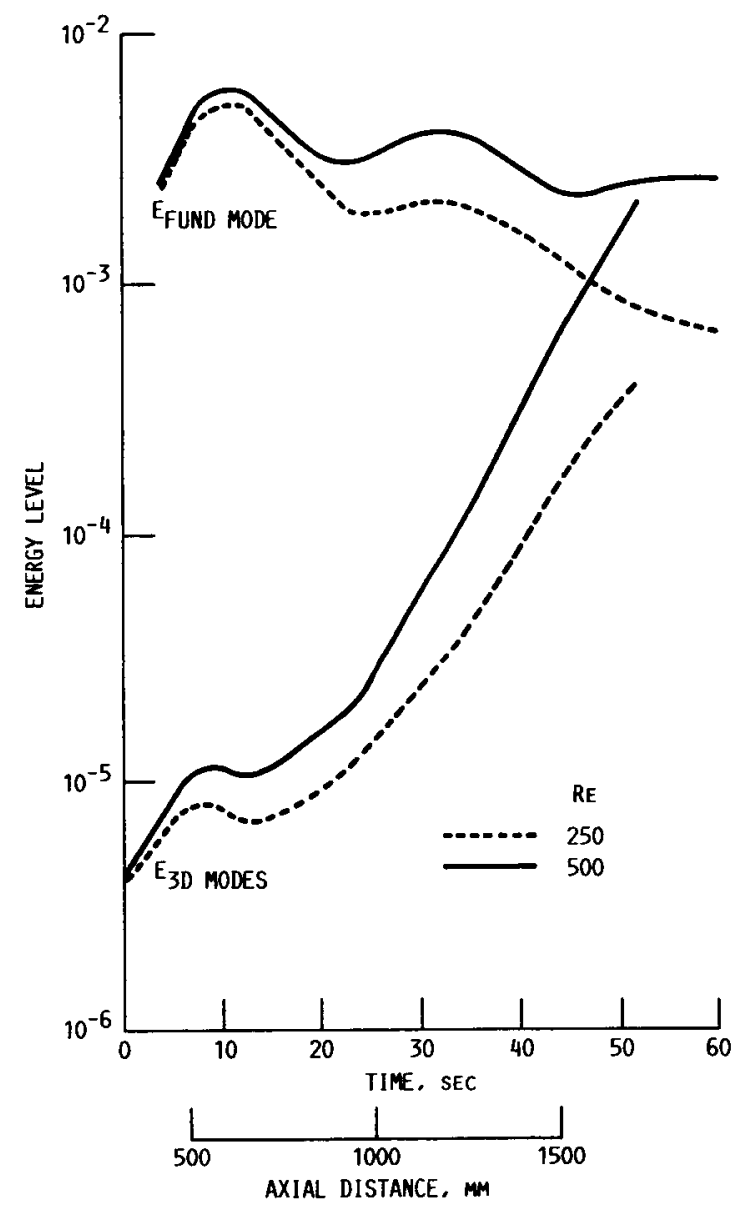

FIGURE 8, - ENERGY CONTENT OF VARIOUS MODES IN THE DNS CALCULATIONS FOR TWO DIFFERENT REYNOLDS MUMBERS ( $64 \times 64 \times 64$ GRID POINTS). 


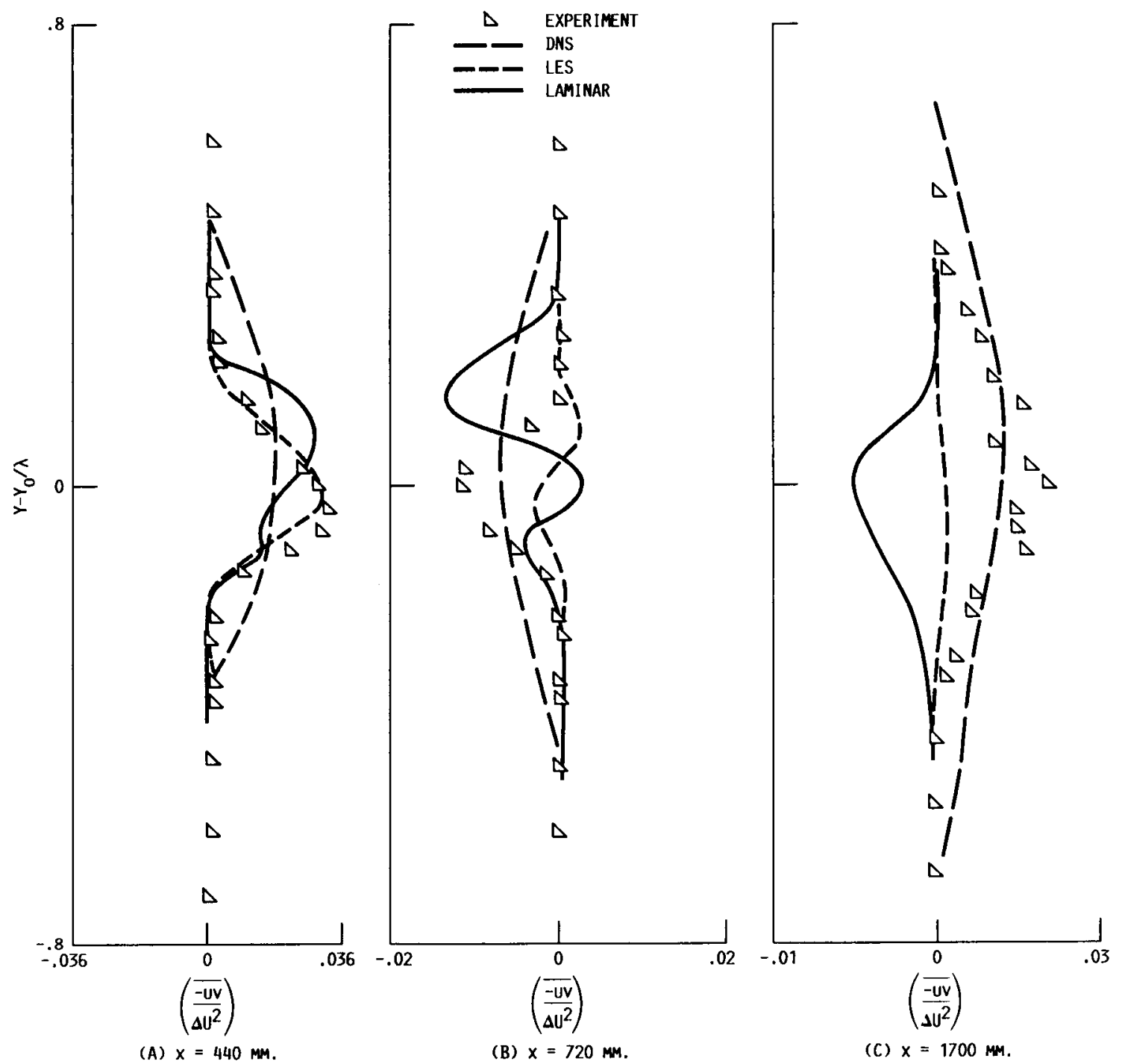

FIGURE 9. - COMPARISON OF CALCULATED AND EXPERIMENTALLY MEASURED REYMOLDS STRESSES AT SEVERAL AXIAL LOCATIONS. 


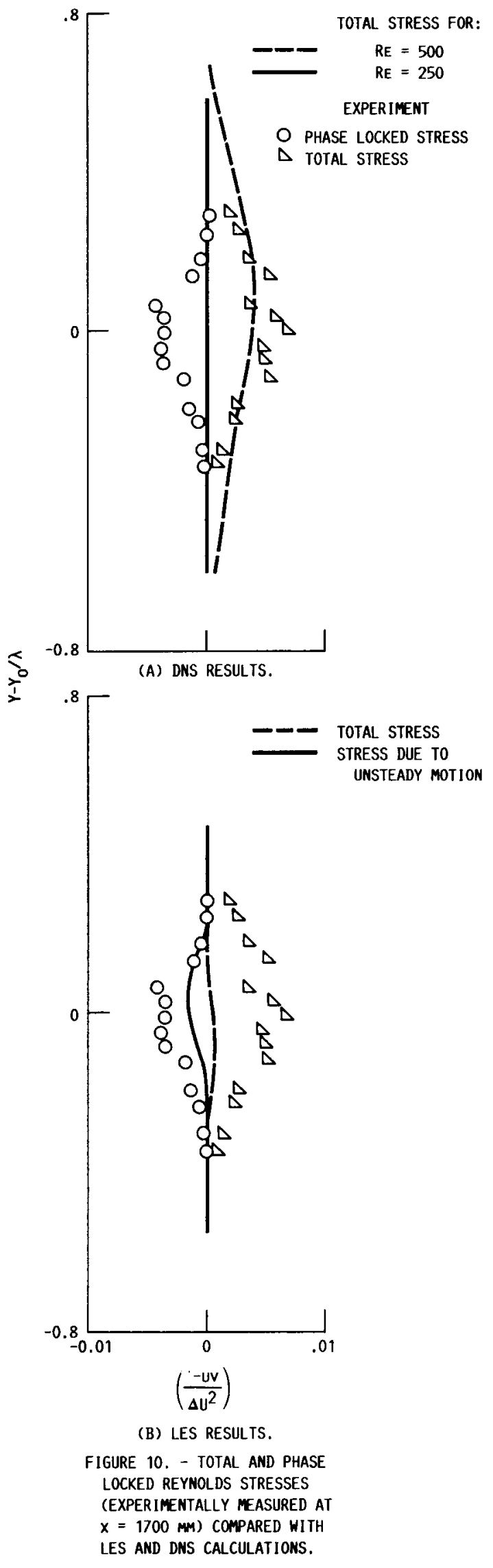




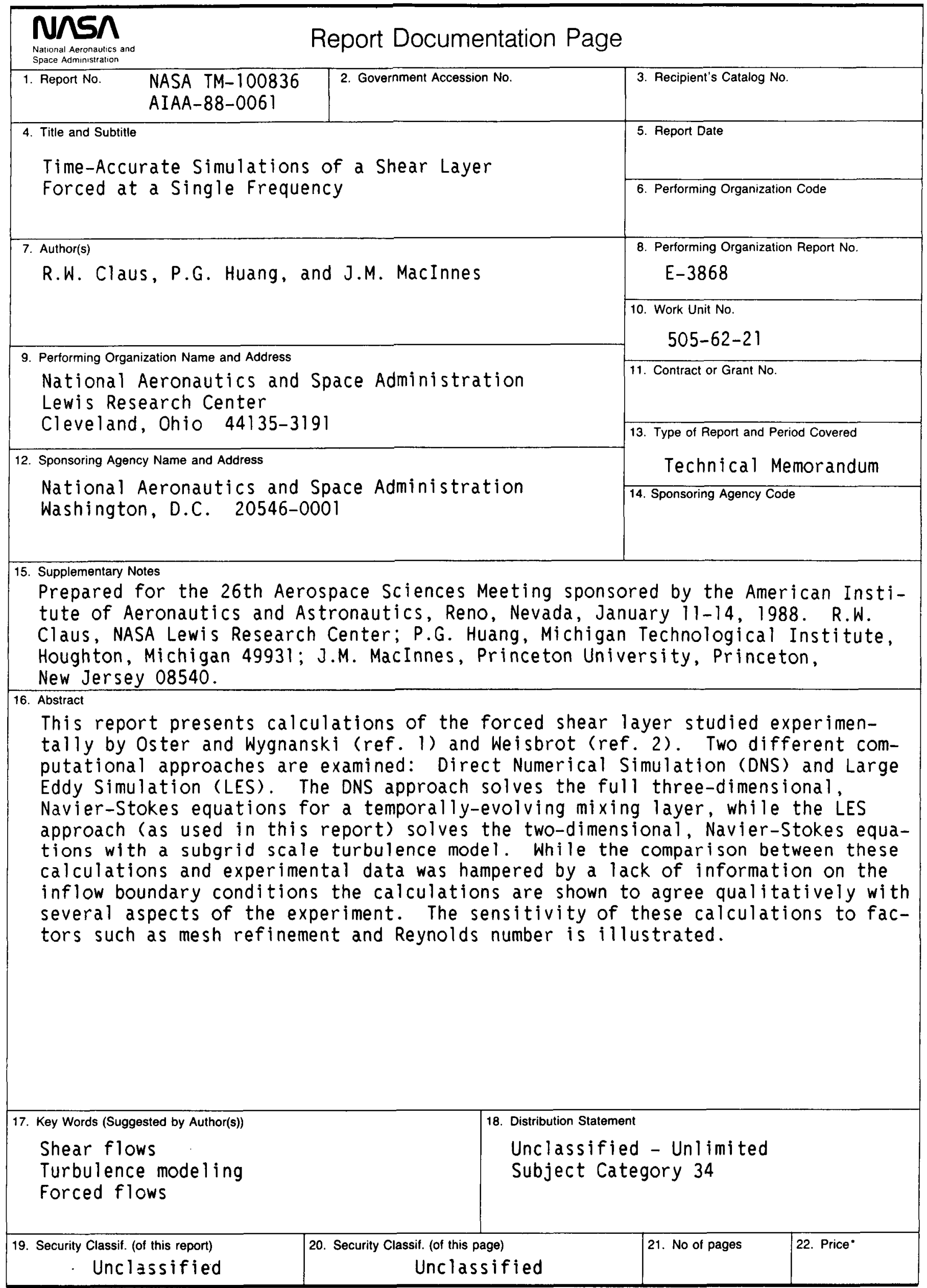

